\title{
260 Anatomy of the submandibular gland
}

A The superficial lobe is larger than the deep lobe.

B Hyoglossus muscle divides deep from superficial lobe.

C The duct opens laterally in the floor of the mouth opposite the second molar tooth.

D The mandibular division of the facial nerve lies superficial to the capsule of the gland.

E The facial artery crosses the gland.

\section{The infratemporal fossa}

A Lies below the posterior cranial fossa.

B Has no anatomical floor.

C Posterior boundary is the styloid apparatus and carotid sheath.

D The maxillary artery and maxillary nerve pass through it.

E Contains the pterygoid venous plexus.

\section{The temporomandibular joint}

A Is a synovial joint.

$B$ Allows both gliding and hinge movements.

C The medial pterygoid muscle inserts into the articular disc.

D Jaw opening is initiated by contraction of the lateral pterygoid muscle.

E Nerve supply is from the maxillary division of the trigeminal. 